\title{
Thermoacoustic Imaging and Therapy Guidance based on Ultra-short Pulsed Microwave Pumped Thermoelastic Effect Induced with Superparamagnetic Iron Oxide Nanoparticles
}

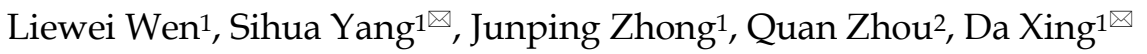 \\ 1. MOE Key Laboratory of Laser Life Science \& Institute of Laser Life Science, College of Biophotonics, South China Normal University, Guangzhou, China; \\ 2. Medical Imaging Center, First Affiliated Hospital of Jinan University, Guangzhou, China. \\ $\triangle$ Corresponding authors: Da Xing, Ph.D. E-mail: xingda@scnu.edu.cn; Sihua Yang, Ph.D. E-mail: yangsh@scnu.edu.cn \\ (c) Ivyspring International Publisher. This is an open access article distributed under the terms of the Creative Commons Attribution (CC BY-NC) license \\ (https://creativecommons.org/licenses/by-nc/4.0/). See http://ivyspring.com/terms for full terms and conditions.
}

Received: 2016.10.08; Accepted: 2017.03.23; Published: 2017.05.11

\begin{abstract}
Multifunctional nanoparticle-mediated imaging and therapeutic techniques are promising modalities for accurate localization and targeted treatment of cancer in clinical settings. Thermoacoustic (TA) imaging is highly sensitive to detect the distribution of water, ions or specific nanoprobes and provides excellent resolution, good contrast and superior tissue penetrability. TA therapy is a potential non-invasive approach for the treatment of deep-seated tumors. In this study, human serum albumin (HSA)-functionalized superparamagnetic iron oxide nanoparticle (HSA-SPIO) is used as a multifunctional nanoprobe with clinical application potential for MRI, TA imaging and treatment of tumor. In addition to be a MRI contrast agent for tumor localization, HSA-SPIO can absorb pulsed microwave energy and transform it into shockwave via the thermoelastic effect. Thereby, the reconstructed TA image by detecting TA signal is expected to be a sensitive and accurate representation of the HSA-SPIO accumulation in tumor. More importantly, owing to the selective retention of HSA-SPIO in tumor tissues and strong TA shockwave at the cellular level, HSA-SPIO induced TA effect under microwave-pulse radiation can be used to highly-efficiently kill cancer cells and inhibit tumor growth. Furthermore, ultra-short pulsed microwave with high excitation efficiency and deep penetrability in biological tissues makes TA therapy a highly-efficient anti-tumor modality on the versatile platform. Overall, HSA-SPIO mediated MRI and TA imaging would offer more comprehensive diagnostic information and enable dynamic visualization of nanoagents in the tumorous tissue thereby tumor-targeted therapy.
\end{abstract}

Key words: thermoacoustic, human serum albumin, superparamagnetic iron oxide nanoparticles, ultra-short pulsed microwave, tumor theranostic.

\section{Introduction}

Recent advances in material science and nanotechnology have promoted the development of new tumor imaging and therapeutic techniques. Multifunctional nanomaterial-mediated fluorescence imaging [1-3], photoacoustic imaging [4-6], magnetic resonance imaging (MRI) [7-9], positron emission computed tomography (PET) imaging [10-12] and X-ray computed tomography (CT) imaging $[13,14]$ for more precise diagnosis of tumors have attracted ever-increasing attention. In addition, emerging nanomedicine in cancer therapy such as photothermal $[1,15,16]$, photoacoustic $[4,6,17]$, photodynamic [18-20], magnetic thermal [9, 21, 22], ultrasonic hyperthermia $[23,24]$, radiation therapy $[25,26]$ and microwave thermal therapy [27] have evoked great interest in their potential clinical application for 
cancer diagnostics and therapeutics research. Overall, multifunctional nanoparticle-mediated imaging and therapeutic techniques are likely to confer excellent leverage in improving precise diagnosis and targeted anticancer therapy.

However, current imaging techniques based on nanoparticles have their own deficiencies, such as low resolution, low sensitivity for small lesions and their side effects on health. Furthermore, emerging nanomedicine technologies such as nanoparticles mediated phototherapy is restricted by limited tissue-penetrability of laser, which particularly poses a challenge to treatment of deep-seated tumors. Although alternating magnetic field (AMF) or microwave-induced hyperthermia therapy offers several advantages including deep penetration in biological tissues when compared to conventional treatment methods, thermal injury to anatomically contiguous healthy tissues is a significant hazard. It is highly desirable to develop a novel approach of multifunctional nanoparticle-mediated tumor imaging and therapy in a versatile platform.

A new theranostic technique based on microwave-pulse induced thermoacoustic (TA) effect is a promising approach for tumor imaging and therapy. TA effect is based on the thermoelastic expansion mechanism, in which microwaveabsorbing agents convert microwave-pulse energy into heat, and subsequently induces shockwave due to rapid thermal expansion [28, 29]. These TA shockwaves can be acquired by ultrasound transducer and reconstructed to form TA images. In addition to inheriting the advantages of microwave imaging, TA imaging technique also provides excellent resolution and deep tissue penetrability, because it is based on ultrasonic propagation and detection [28, 30, 31]. More importantly, we have proven that the powerful shockwave induced with single wall carbon nanotubes when pumped by pulsed microwave could destroy mitochondria of cancer cells and thus highly-efficiently inhibit tumor growth [32]. It inspires us to further explore the potential application of TA effect as a novel theranostic technique.

Based on the above, human serum albumin (HSA)-functionalized superparamagnetic iron oxide nanoparticles (HSA-SPIO) is developed to be a multifunctional nanoprobe for MRI, TA imaging and therapy. HSA as the most abundant plasma protein is completely biocompatible, easily produced and lacks toxicity and immunogenicity $[33,34]$, which has been proven to be useful in improving the circulation half-life and accumulation of the drug molecule at tumor site [35]. In addition, superparamagnetic iron oxide nanoparticles (SPIO) as ideal MRI contrast agents have been approved for clinical usage [36]. Of note, SPIO was shown to have resonance in the microwave region $[37,38]$. SPIO-mediated microwave hyperthermia offers possibilities for targeted treatment [27], and shows potential for being a TA imaging contrast agent [39-41]. In this study, HSA-SPIO as a $\mathrm{T}_{2}$ contrast agent in MRI enables clear delineation of the tumor. Besides, HSA-SPIO pumped by microwave-pulse is able to generate strong TA shockwave, which can be detected and reconstructed to form TA images for sensitively and accurately indicating the HSA-SPIO accumulation and TA effect enhancement in tumor region, thereby providing a noninvasive imaging-guided approach for the subsequent TA therapy. HSA-SPIO mediated dual-imaging approach would be expected to provide more comprehensive and complementary information of tumor than single imaging mode, in which MRI provides clear anatomical images and TA imaging provides deep tissue information of tumor. More importantly, owing to the ultra-short pulsed microwave pumped powerful TA shockwave at cellular level and deeper penetrability than that of laser in biological tissues, TA therapy shows an excellent anti-tumor effect for the deep-seated tumor, and have broader application prospects than phototherapy. Overall, HSA-SPIO consists of two clinically approved components and shows potential for development as a nanotheranostic agent for MRI and TA imaging guided TA therapy on the versatile microwave platform in the future clinical application.

\section{Results}

The HSA-SPIO was prepared according to Scheme 1A. HSA-SPIO was intravenously injected into mice, and the MRI provided tumor localization image. Real-time TA imaging was used to indicate HSA-SPIO accumulation and TA effect enhancement in tumor region (Scheme 1B). Finally, high doses of nanoparticles were injected into mice and irradiated with high energy microwave-pulse after accumulation of HSA-SPIO at tumor site. Integrating the advantages of high excitation efficiency and deep penetrability of ultra-short pulsed microwave, as well as the long half-life and targeted accumulation of HSA-SPIO in tumor, ultra-short pulsed microwave pumped HSA-SPIO could generate powerful TA shockwave thus cause mechanical destruction of cancer cells and inhibit tumor growth (Scheme 1C). 
A

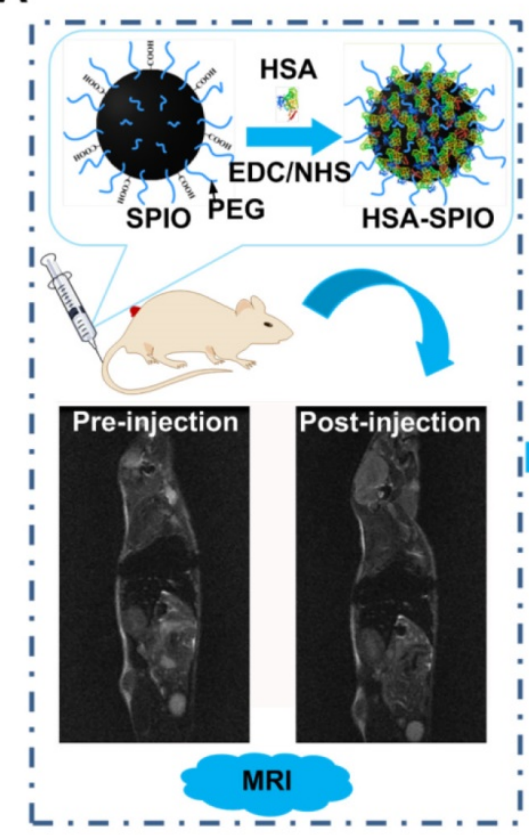

B

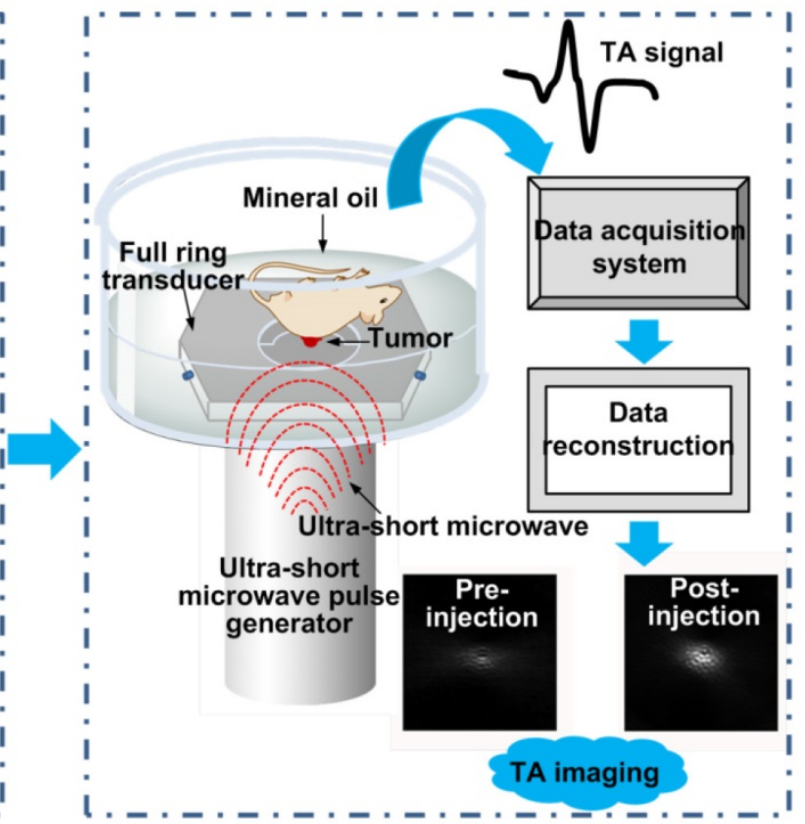

C

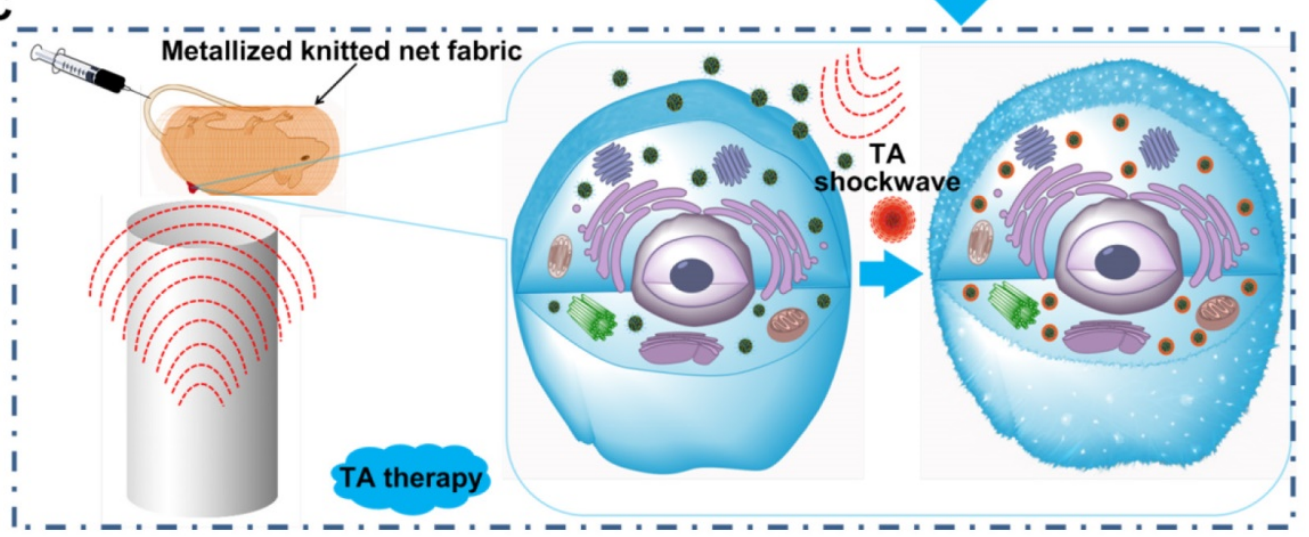

Scheme 1. (A) Schematic illustration of the process of HSA-SPIO synthesis and its intravenous injection into mice for MRI. (B) Schematic illustration of the TA imaging system. (C) Schematic illustration of HSA-SPIO as a TA therapeutic agent for tumor therapy.

\section{Characterization of SPIO and HSA-SPIO}

The monodisperse SPIO was modified with HSA to enhance the stability and biocompatibility of SPIO. Transmission electron microscopy (TEM) images showed that the SPIO was spherical shaped with a diameter of about $200 \mathrm{~nm}$ (Fig. 1A). After functionalized with HSA, a nearly transparent thin layer of HSA could be observed on the surface of SPIO due to the low electron density (Fig. 1B and insert). The average hydrodynamic diameter of SPIO was 183 $\pm 11 \mathrm{~nm}$ as assessed by dynamic light scattering (DLS) (Fig. 1C), while the HSA-SPIO increased to $236 \pm 26$ $\mathrm{nm}$ due to the hydration shell on the protein surface. On the other hand, the zeta potential of SPIO decreased from $-4.5 \pm 0.5 \mathrm{mV}$ to $-23.8 \pm 0.4 \mathrm{mV}$ after coated with HSA (Fig. 1D). The increased size and decreased surface charge indicated that the HSA was successfully modified on the SPIO surface. The presence of HSA on the SPIO surface was further confirmed by Fourier transform infrared (FT-IR) spectroscopy (Fig. 1E). The absorption bands at 1640 $\mathrm{cm}^{-1}$ and $1396 \mathrm{~cm}^{-1}$ were associated with carboxylate groups in SPIO; $580 \mathrm{~cm}^{-1}$ and $430 \mathrm{~cm}^{-1}$ were attributed to Fe-O vibration, which was observed in SPIO and HSA-SPIO [42]. Meanwhile, the characteristic bands of HSA at $1648 \mathrm{~cm}^{-1}$ (amide I), and $1540 \mathrm{~cm}^{-1}$ (amide II) also appeared and weakened in HSA-SPIO. These results confirmed that HSA has been modified on SPIO through the formation of amide bond between $-\mathrm{COOH}$ of SPIO and $-\mathrm{NH}_{2}$ of HSA. As we expected, HSA was able to improve the colloidal stability of SPIO in a physiological environment. Even after 8 hours, HSA-SPIO remained stably dispersed in PBS, PRMI-1640 and serum. In contrast, pure SPIO showed agglomeration and settlement (Fig. S1). 
A

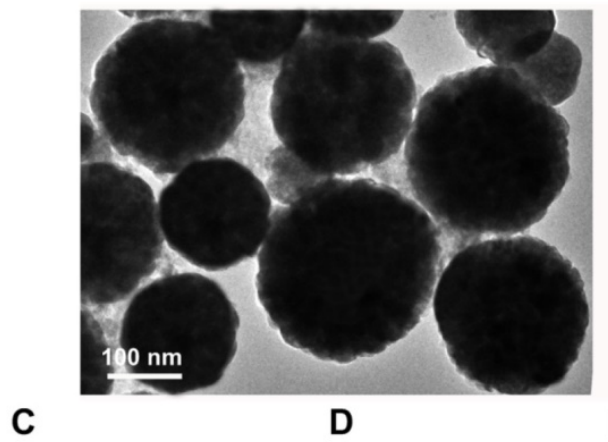

B
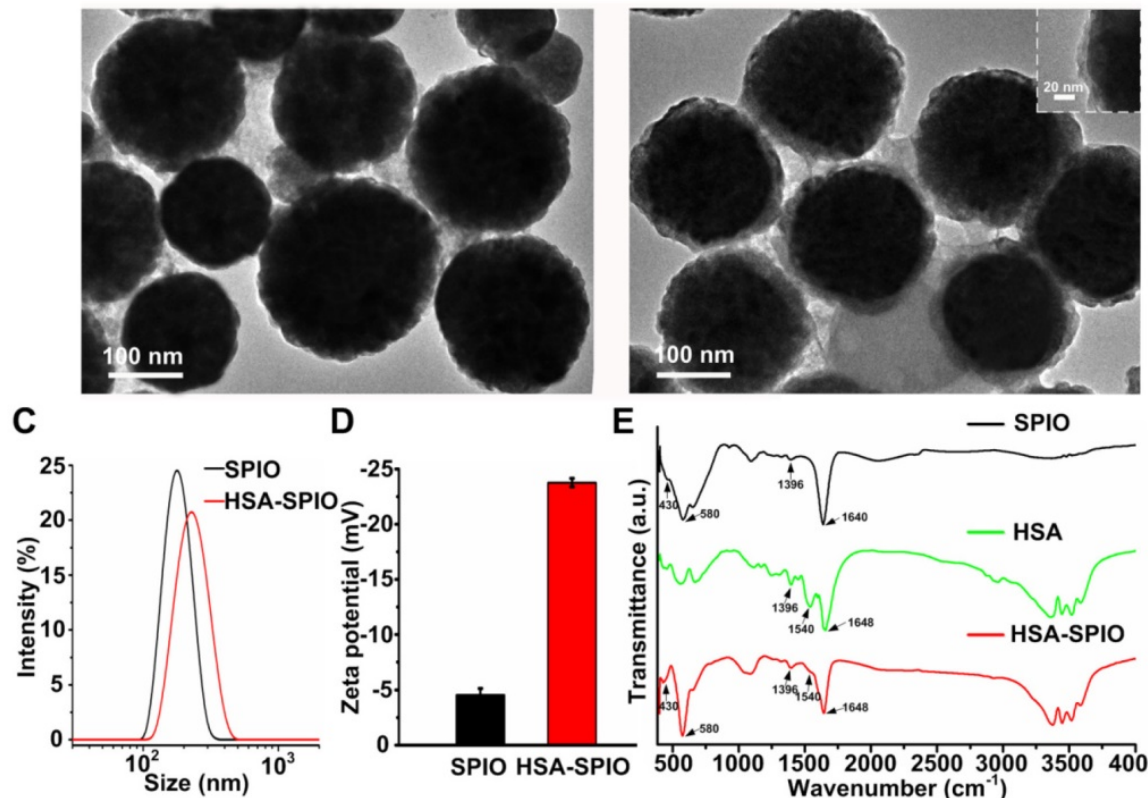

E

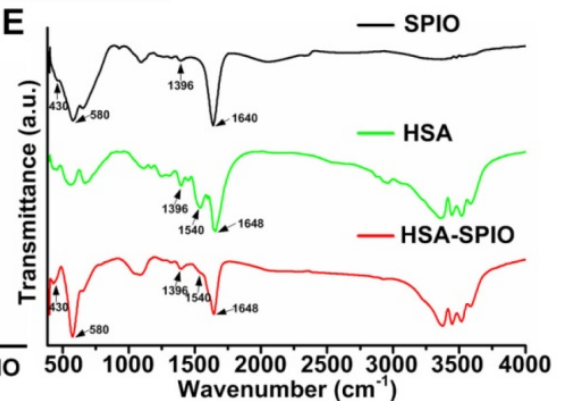

Figure 1. Characterization of SPIO and HSA-SPIO. (A) TEM images of SPIO and (B) HSA-SPIO. (Inset is HR-TEM). (C) Particle size of SPIO and HSA-SPIO determined by DLS. (D) Zeta potential change of SPIO and HSA-SPIO. (E) FT-IR spectra of SPIO, HSA and HSA-SPIO.

\section{TA/MR properties of HSA-SPIO}

To evaluate the capability of HSA-SPIO as a nanotheranostic agent for microwave-induced TA imaging and therapy, we firstly detected the TA signals of HSA-SPIO at various concentrations (Fig. 2A). The TA signal intensity enhanced with the increase of concentration, and showed a linear relationship with HSA-SPIO concentrations (Fig. 2B). This result suggested that HSA-SPIO possessed microwave absorption property [39, 40] and could generate TA shockwave under ultra-short pulsed microwave excitation. Then the TA imaging contrast enhancement ability of HSA-SPIO was verified by TA images of plastic tubes (diameter: $2 \mathrm{~mm}$ ) filled with deionized water and different concentration of HSA-SPIO (SPIO 0.05, 0.1, 0.2, 0.4, and $0.8 \mathrm{mg} \mathrm{mL}^{-1}$ ). TA imaging contrast was obviously enhanced with increase in the concentration (Fig. 2C). Moreover, mean gray of each sample represented signal intensity, which showed a linear correlation with the HSA-SPIO concentration (Fig. 2D). Given that both water and ion in tissues could contribute to TA imaging contrast enhancement $[43,44]$, the TA images of HSA-SPIO dispersed in physiological saline buffer (PBS) to simulate nanoparticles accumulation region were also acquired. Similarly, it showed contrast enhancement with the increase in the concentration of HSA-SPIO (Fig S2A, B). In addition, the absorption coefficient of biological tissue is about $3.8 \sim 28 \mathrm{~m}^{-1}$ at $434 \mathrm{MHz}$; the penetration depth of ultra-short pulsed microwave was up to $26.2 \mathrm{~cm}$ for fat, bone, and tissues with low water content, while for muscle skin and tissues with high water content, its penetration depth is about $3.57 \mathrm{~cm}$ [45]. Thereupon, in a soft tissue phantom, TA image of HSA-SPIO solution in a plastic tube embedded about $1 \mathrm{~cm}$ from the surface of muscle was acquired. TA contrast within the red dotted circle where HSA-SPIO embedded inside showed obvious enhancement (Fig S3A). The average TA signals within the red dotted circle showed $\sim 2$-4-fold improvement compared to those of the surrounding muscle tissues (Fig S3B). This implied that HSA-SPIO could serve as an effective TA imaging contrast agent. Since, SPIO is also a $T_{2}$ contrast agent for MRI, the superparamagnetism of HSA-SPIO was evaluated. The homogeneously dispersed HSA-SPIO showed a fast response to the external magnetic field (Fig. S4 insert). As showed in saturation magnetization curves, the saturation magnetization value $(M s)$ of HSA-SPIO was $71.3 \mathrm{emu} \mathrm{g}^{-1}$ lower than that of SPIO (79.8 emu g-1 ${ }^{-1}$, because the HSA on the SPIO reduced the total ferrite content in HSA-SPIO. Neither SPIO nor HSA-SPIO showed hysteresis loops, and both of these possessed strong superparamagnetism (Fig. S4). Then, the relaxation rate $\left(1 / \mathrm{T}_{2}\right)$ of HSA-SPIO was measured by a medical $1.5 \mathrm{~T}$ MRI instrument. MRI signals were significantly decreased with increase in iron concentration, as showed in the $\mathrm{T}_{2}$-weight MRI (Fig. S5A). The transverse relaxivity $\left(r_{2}\right)$ was $53.7 \mathrm{mM}^{-1}$ $\mathrm{s}^{-1}$ (Fig. S5B), which revealed its good capability to be a $T_{2}$ negative contrast agent. Above all, these results indicated that HSA-SPIO could serve as a dual mode contrast agent for both MRI and TA imaging. 

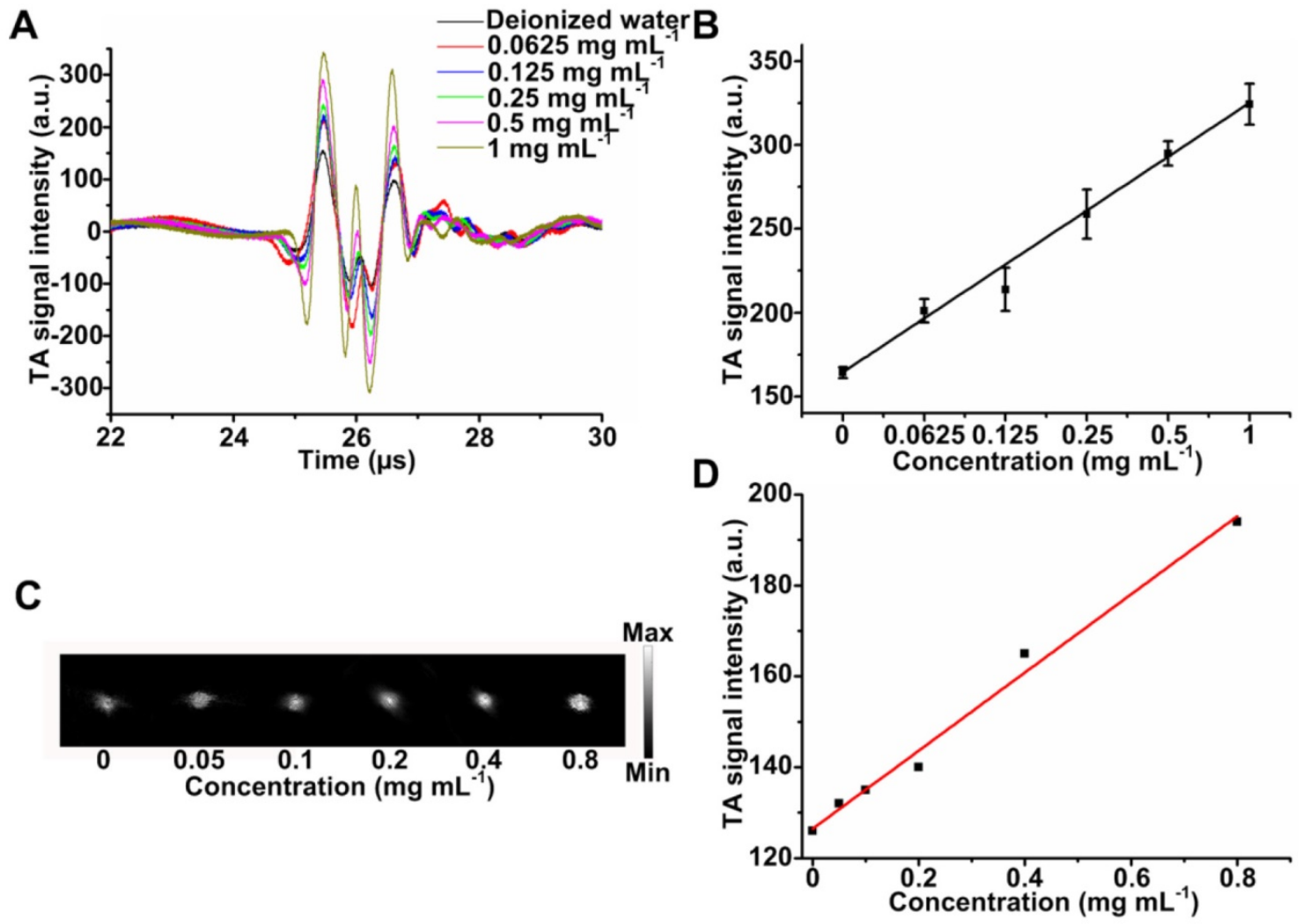

Figure 2. TA properties of HSA-SPIO. (A) Microwave-pumped TA signal of HSA-SPIO at various concentrations. (B) Linear fit of TA signal intensity in corresponding concentrations. (C) TA tomography imaging of HSA-SPIO solution at different concentrations. (D) Linear fit of TA signal intensity.

\section{Cytotoxicity and hemolysis assay}

Before intravenous injection with HSA-SPIO, we further confirmed its biocompatibility by evaluating the cytotoxic characteristics and hemolysis rate in vitro. Different concentrations $(0.0625,0.125,0.25,0.5$, 1, $2 \mathrm{mg} \mathrm{mL}^{-1}$ ) of HSA-SPIO were incubated with $4 \mathrm{~T} 1$ cells for 24 hours or 48 hours. The cells retained their viability even on incubation with $2 \mathrm{mg} \mathrm{mL}^{-1}$ of HSA-SPIO for 48 hours (Fig. S6A). The hemolytic behavior of HSA-SPIO at different concentrations $\left(0.0625-2 \mathrm{mg} \mathrm{mL}^{-1}\right)$ as well as that of the negative control exhibited negligible hemolysis; all $\mathrm{HR} \%$ were below 1\% (Fig. S6B). Except for that in the positive control group, no visible broken erythrocyte was observed (insert of Fig. S6B), which indicated that HSA-SPIO did not cause erythrocyte membrane damage. Therefore, HSA-SPIO showed good biocompatibility and safety for in vivo imaging and therapy.

\section{In vivo MRI/TA imaging of tumor model}

For tumor therapy, it is vital to accurately detect tumor region, and ensure accumulation of the nanoagents in the entire tumor mass. Herein, HSA-SPIO ( $\left.1 \mathrm{mg} \mathrm{mL}^{-1}, 0.1 \mathrm{~mL}\right)$ as a $\mathrm{T}_{2}$ contrast agent of MRI was intravenously injected into the mice bearing subcutaneous 4T1 breast tumors. Sagittal plane images of mice were acquired before and after administration of nanoparticles (Fig. 3A). The red circles indicated the area where the tumor was located. After intravenous injection of HSA-SPIO, the MRI signal in tumor regions was obviously decreased. At 4 hours post-injection, the tumor region appeared noticeable darkened, which indicated a large accumulation of HSA-SPIO in the tumor. The quantified $\mathrm{T}_{2}$-weighted MRI signal of tumor also showed a gradual decrease. Between 4 and 24 hours post-injection, the tumor MRI signal recovered to some extent, which suggested that the HSA-SPIO was gradually cleared from the tumor (Fig. 3B). Therefore, the MRI images showed tumor localization and indicated the dynamic distribution of HSA-SPIO in tumor. Furthermore, TA imaging of tumor also showed obvious TA contrast enhancement at 4 hours after injection (Fig. 3C). The average TA signals in the tumor region were significantly increased (Fig. 3D), which was consistent with the MRI results. The enhancement of TA signals was attributable to HSA-SPIO accumulation and retention in tumor at 4 hours after injection. This time point was critical for subsequent microwave induced TA therapy. The above findings indicated that HSA-SPIO could be used as an MRI contrast agent for delineation of the 
tumor, while it also served as TA imaging contrast agent to indicate the TA effect enhancement in tumor. In addition, the distribution of HSA-SPIO was further confirmed by Prussian blue staining which showed positive staining for iron. The major organs and tumor were collected at 4 hours after intravenous injection with nanoparticles. In the tumor slice, many staining spots could be observed, which showed rich accumulation of HSA-SPIO in the tumor. It was correlated well with the in vivo MRI/TA imaging results (Fig. S7A). The Fe levels of major organs and tumors were measured by ICP-MS at 4 hours post-injection. The Fe levels in tumor tissue were up to $\sim 22 \% \mathrm{ID} \mathrm{g}^{-1}$ which is attributable to HSA-SPIO accumulation. While the high Fe levels of liver and spleen were mainly attributed to the uptake of nanoparticles by macrophages in reticuloendothelial systems (RES) (Fig. S7B). The capability of TA imaging contrast enhancement and high tumor accumulation inspired us to use HSA-SPIO for TA therapy.

\section{Evidence of TA shockwave destruction and in vitro cancer cell viability test}

In order to evaluate the destructive effect of TA shockwave, HSA-SPIO was enwrapped with a cell-like alginate-polylysine-alginate microcapsule [46] (Fig. S8A). Irradiation with ultra-short pulsed microwave broke the microcapsules (Fig. S8C). In contrast, microcapsules without HSA-SPIO maintained good morphology (Fig. S8B) even irradiated with microwave for $10 \mathrm{~min}$ (Fig. S8D). These results implied that the HSA-SPIO under microwave-pulse excitation could generate a powerful TA shockwave that destroyed the membrane. Thereafter, the killing effect of TA shockwave for cancer cells was tested. Firstly, HSA-SPIO was labeled with FITC (FITC-HSA-SPIO) and incubated with 4T1 cells for different durations of time. Low fluorescence of FITC-HSA-SPIO was observed on the cell membrane after incubation for 1 hour. Two hours later, bright fluorescence was observed in the membrane and cytoplasm (Fig. S9), indicating the accumulation of FITC-HSA-SPIO, and which is likely related to the interaction of HSA sheath with cell surface glycoprotein receptor or SPARC. Cell membranes permeability is known to increase with initiation of cell injury. The increase in cell membranes permeability would allow the translocation of fluorescent dyes into the nucleus [47]. In this study, propidium iodide (PI) as a qualitative indicator of the cell plasma membrane integrity was used for assessing the loss of cell membrane integrity caused by the TA shockwave. PI could not penetrate viable cells, while it was able to penetrate comparatively leaky membranes [48]. After incubation with HSA-SPIO for 2 hours, PI was added and immediately irradiated with microwave for 5 min. In the microwave-alone or HSA-SPIO-alone treatment groups as well as in the control group, the cells retained their morphology, and no fluorescence was observed in the nucleus. In contrast, in the HSA-SPIO+microwave group, 4T1 cells displayed red fluorescence in nucleus, which indicated that the enhanced membrane permeability allowed PI infiltration into cells (Fig S10). It implied that the powerful TA shockwave of HSA-SPIO impaired the cell membrane integrity while the cell membrane damage was related to cell death [49]. Subsequently, cell viability after treatment with microwave and various concentrations of nanoparticles was assessed by Cell Counting Kit-8 (CCK-8) assay. After 24 hours of incubation with HSA-SPIO, the relative viability of cells sharply decreased with the increase in the concentration of HSA-SPIO on irradiation with microwave (Fig. 4A). To further visually identify the cell killing capability of TA effect, 4T1 cells were incubated with HSA-SPIO $\left(0.2 \mathrm{mg} \mathrm{mL}^{-1}\right)$ and irradiated with ultra-short pulsed microwave. Six hours later, 4T1 cells were stained with Hoechst 33258 to directly observe the live and death/apoptotic cells. Cells in the HSA-SPIO+microwave group showed more bright blue fluorescence in condensed chromatin of nucleus than that in other groups (Fig. 4B). It indicated that HSA-SPIO under ultra-short pulsed microwave radiation had induced cell death/apoptosis. Finally, flow cytometry assays (FACS) was used for quantitative analysis of the tumor cell killing effect. The apoptotic cells were stained by Alexa Flour 488-annexin V, while the necrotic cells were stained with propidium iodide (PI). Low apoptotic and necrotic rates were observed in cells treated only with nanoparticles or microwave, which showed negligible differences from those in the control group; this further confirmed the safety of the nanoparticles and microwave for cells. Notably, the cells treated with HSA-SPIO and microwave showed a remarkable increase in cell apoptosis and death (Fig. 4C). Statistical analysis suggested that TA effect of HSA-SPIO induced apoptosis of $79 \%$ of the cells (annexin V-FITC positive cells; Fig. 4D), which indicates that the mechanism of TA therapy is mainly due to cell apoptosis rather than necrosis. The above findings proved that HSA-SPIO pumped by ultra-short pulsed microwave could generate powerful TA shockwaves, which mechanically destroys the membrane structure of cells thus showing high-efficacy for causing cancer cell death. 
A
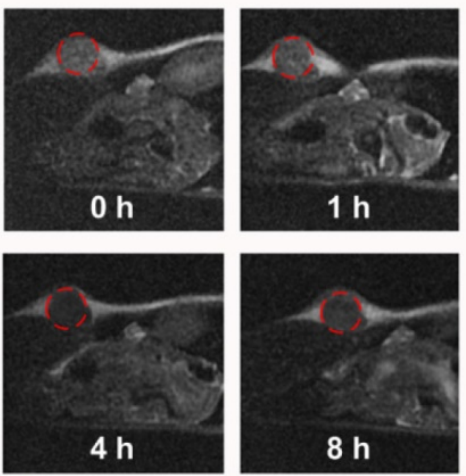

$8 \mathrm{~h}$
B
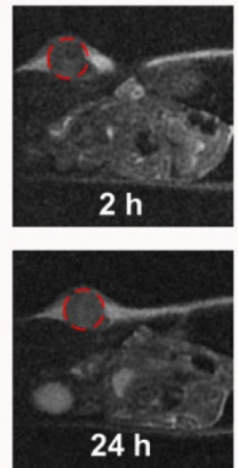

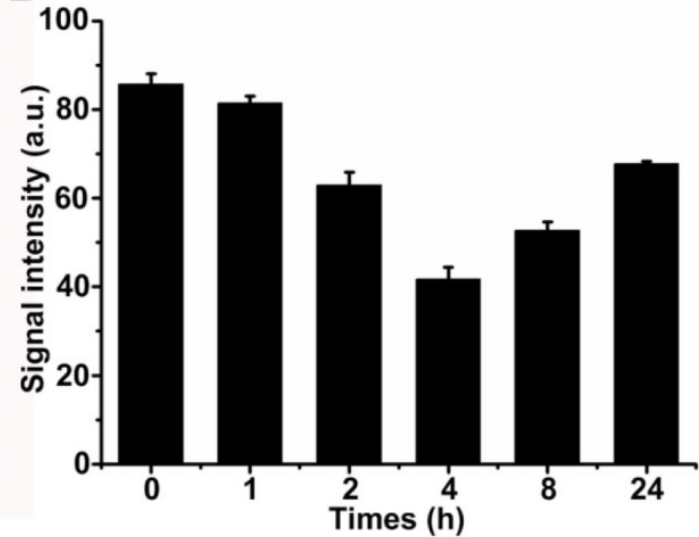

C

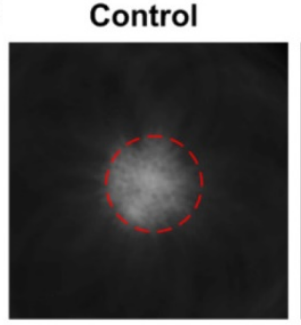

$4 \mathrm{~h}$

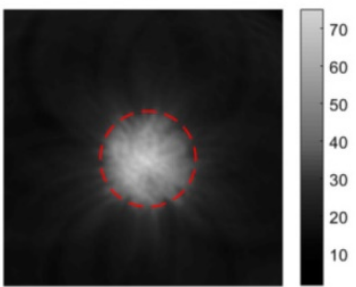

Photograph

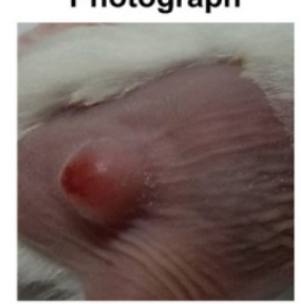

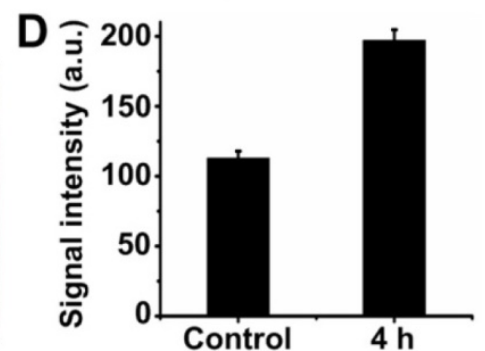

Figure 3. In vivo multimodal imaging. (A) $\mathrm{T}_{2}$-weighted MR images of $4 \mathrm{~T} 1$ tumor-bearing mice after intravenous injection of HSA-SPIO. (B) $\mathrm{T}_{2}$ signal intensity in the tumor region. (C) TA images of mice before HSA-SPIO injection and at $4 \mathrm{~h}$ post-injection. (D) TA signal intensity of tumor region.

A

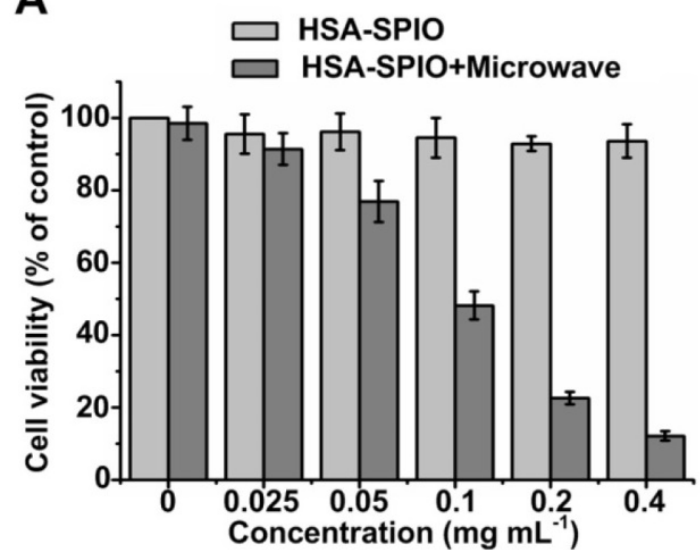

C

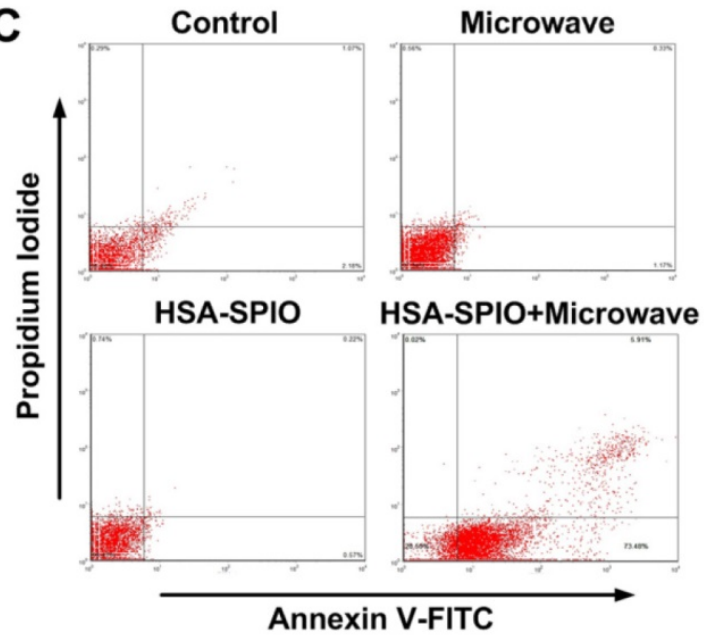

B

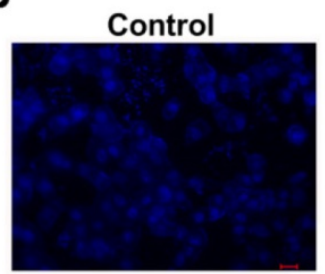

HSA-SPIO

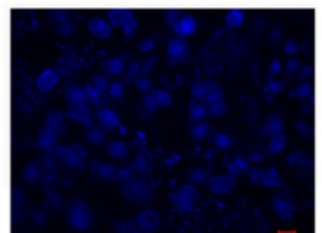

D

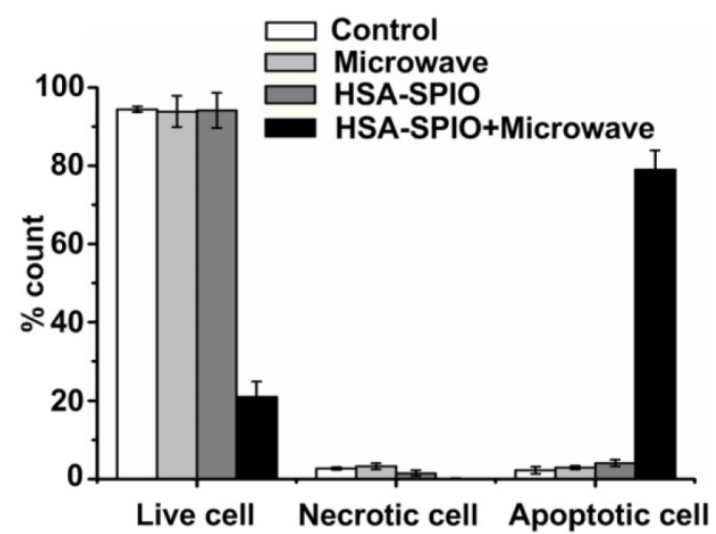

Figure 4. Cytotoxic effect of TA induced by HSA-SPIO. (A) TA effect of HSA-SPIO on cell viability at different concentrations. (B) Fluorescent images of apoptotic tumor cells in different treatment groups on staining with Hoechst 33258. (Scale bar: $20 \mu \mathrm{m}$ ). (C) Cell death analysis by FACS with annexin V-FITC/PI double staining. (D) Statistical analysis of FACS data. 


\section{In vivo TA therapy}

Motivated by the tumor accumulation of HSA-SPIO and its powerful TA effect to highly-efficiently kill cancer cells, in vivo TA therapy was performed in 4T1 tumor-bearing mice. Mice were randomly divided into four groups for different treatments: microwave irradiation, HAS-SPIO injection, no treatment or both microwave irradiation and HAS-SPIO injection. Evaluation of tumor volume showed a remarkable inhibition of tumor growth only in the HSA-SPIO+microwave group, whereas in the other treatment groups, tumor showed a comparable trend of rapid growth (Fig. 5A). The representative mice photos showed the change in tumor size before TA treatment and 21 days later (Fig. 5B), which suggest that neither HSA-SPIO nor microwaves, per se, affect tumor development. Histological analysis of tumor tissue in different treatment groups at two days post-treatment showed almost no damage to tumors in the control, microwave-alone and HSA-SPIO-alone groups. In marked contrast, the tumor tissue sections from the HSA-SPIO+microwave group showed abundant karyorrhectic debris and considerable karyolysis, which indicated a significant damage to cancer cells (Fig. 5C). The tumor slices were further stained by terminal deoxynucleotidyl transferase dUTP nick end labeling (TUNEL) to evaluate the treatment efficacy. Tumors treated with HSA-SPIO and microwave irradiation showed bright fluorescence, which implies remarkable cell apoptosis, while only background level fluorescence was observed in the other treatment groups (Fig. S11). These findings were consistent with the results of H\&E staining. Furthermore, survival curves for the different groups showed that life span of mice in the control, HSA-SPIO-alone and microwave-alone groups were not more than 34 days (Fig. 5D). However, mice in the HSA-SPIO+microwave group survived for over 50 days.

B

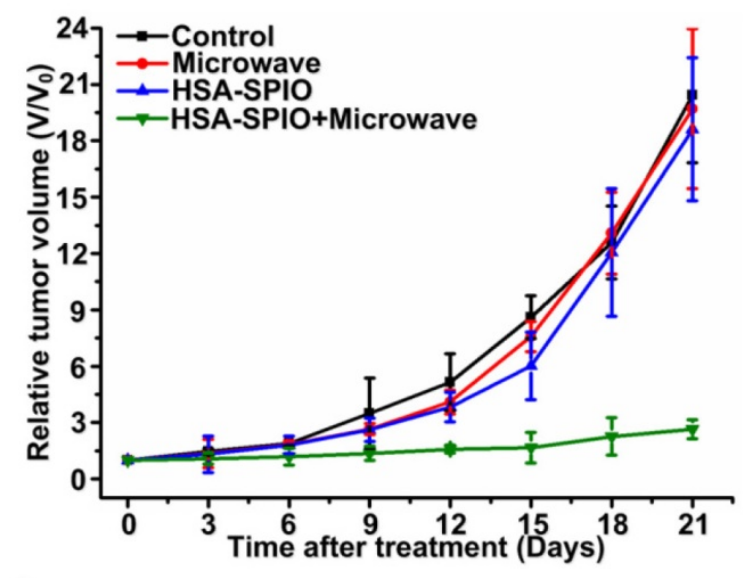

C
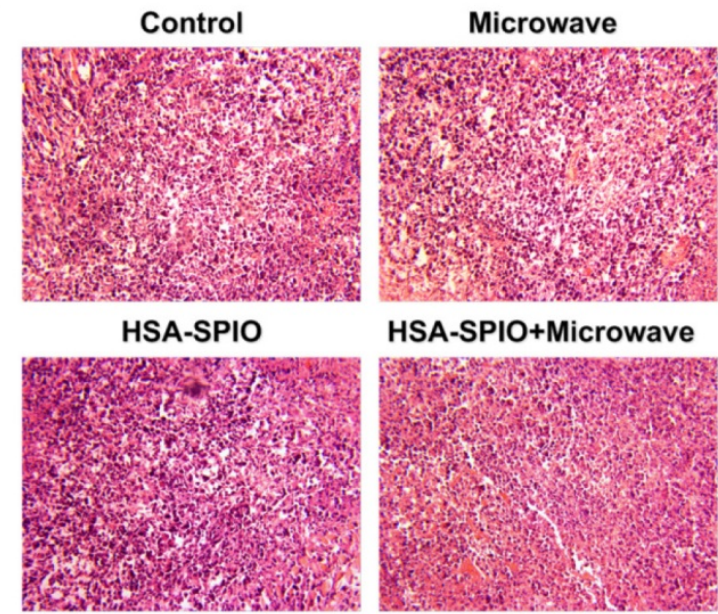

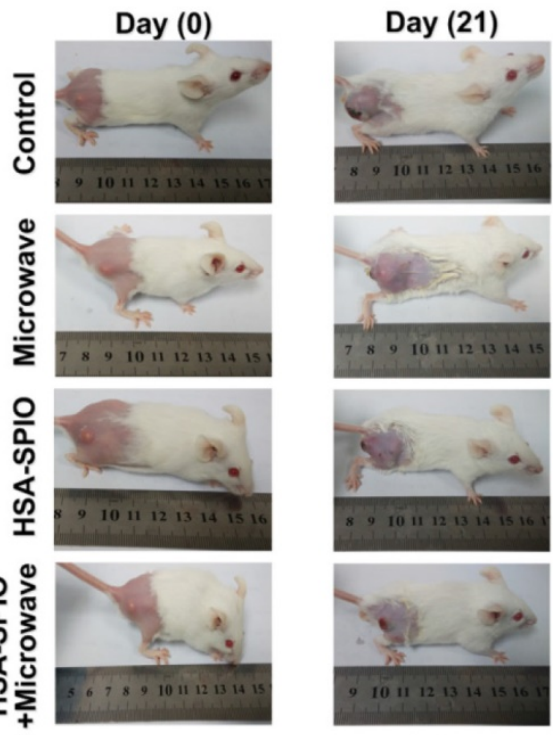

D

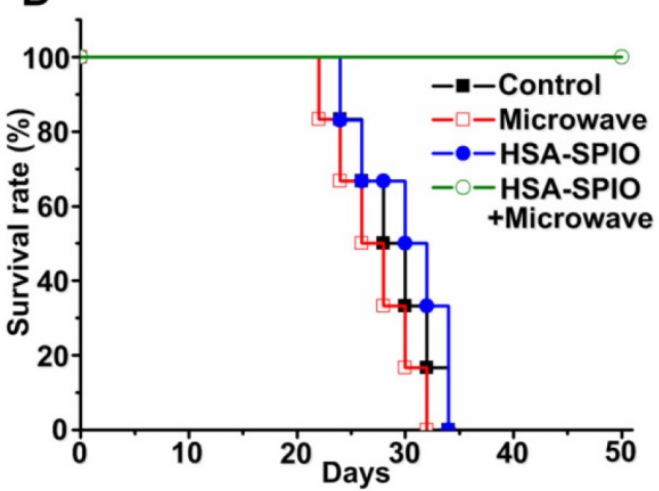

Figure 5. TA therapy in subcutaneous xenograft tumor model. $(A)$ Relative volumetric change in tumor size in various treatment groups $\left(V_{0}=\right.$ tumor volume at the time of microwave treatment). Data presented as mean $\pm S D(n=6)$; $(B)$ Representative photographic images showing effect of different treatment on tumors preand post-21 days of treatment; (C) Histopathological examination of the excised tumors $48 \mathrm{~h}$ after treatment (H\&E stained representative specimens at $10 \times$ magnification). (D) Survival rate of mice bearing $4 \mathrm{~T} 1$ tumor by study group. 
TA therapy is a recently proposed therapeutic technique, which is different from the microwave thermal therapy. Herein, the temperature change of deionized water and HSA-SPIO solution were recorded by infrared thermal camera before and after microwave irradiation. No more than $\pm 1{ }^{\circ} \mathrm{C}$ temperature change was found in infrared thermal imaging pictures even after microwave irradiation with high concentration of HSA-SPIO $\left(8 \mathrm{mg} \mathrm{mL}^{-1}\right)$ for 10 min (Fig. S12A, B). After intravenous injection of HSA-SPIO, the thermographic images of tumor-bearing mice showed negligible increase in the temperature of the tumor region (Fig. S12C), which indicated that there was just a tiny temperature increase during TA therapy. These results showed that HSA-SPIO mediated TA therapy efficiently inhibited tumor growth, which prolonged the life span of tumor-bearing mice, while its mechanism of action did not depend on hyperthermia.

\section{Analysis of side effects}

HSA has already been approved by FDA [16, 41, 50] and various non-toxic and biodegradable formulations of SPIO are available for using as contrast agents for MRI [33, 51, 52]. However, it was necessary to evaluate the potential side effects of nanoparticles, microwave and TA therapy. Histological analysis of the major organs of mice including heart, liver, spleen, lung, and kidney showed no obvious signs of organ damage or inflammatory lesions in the HSA-SPIO, microwave irradiation or HSA-SPIO+Microwave groups as compared to that in the control group (Fig. 6A). Body weights of mice were monitored during the entire treatment course. No significant difference was observed between the treatment and control groups in this respect (Fig. 6B), which indicated that neither HSA-SPIO nor the therapeutic approach resulted in acute fatal toxicity.
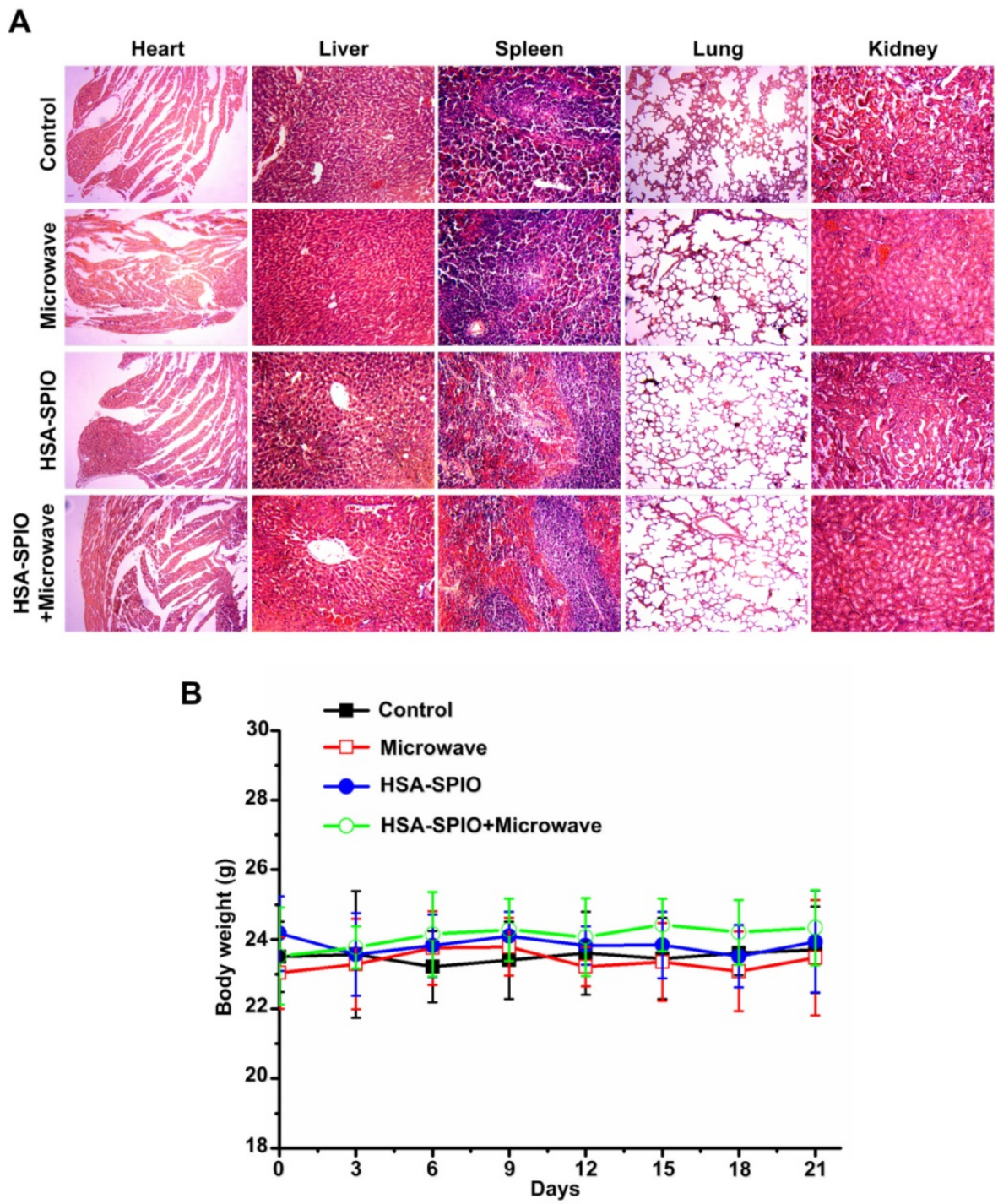

Figure 6. Side effects of TA therapy. (A) Representative H\&E stained sections of major organs (heart, liver, spleen, lung, and kidney) at 10×magnification. (B) Body weights of mice were measured during the 21-day evaluation period under different conditions. Data presented as mean $\pm S D(n=6)$. 


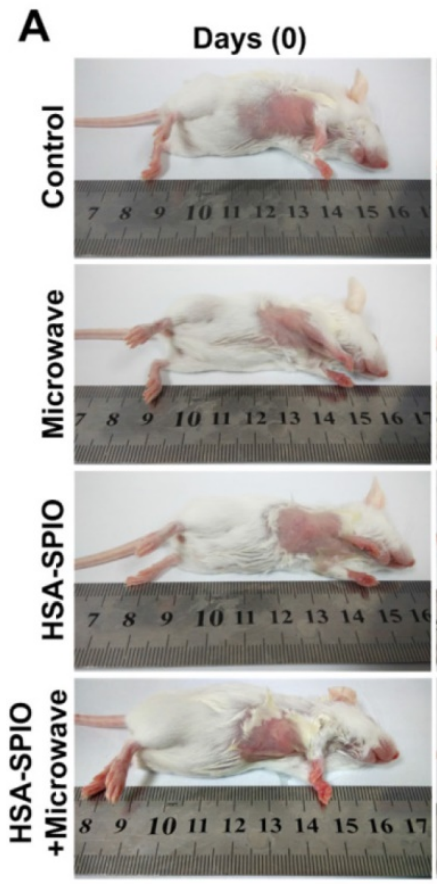

D

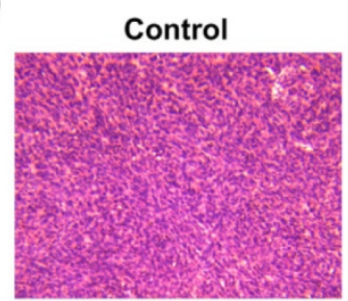

Days (21)

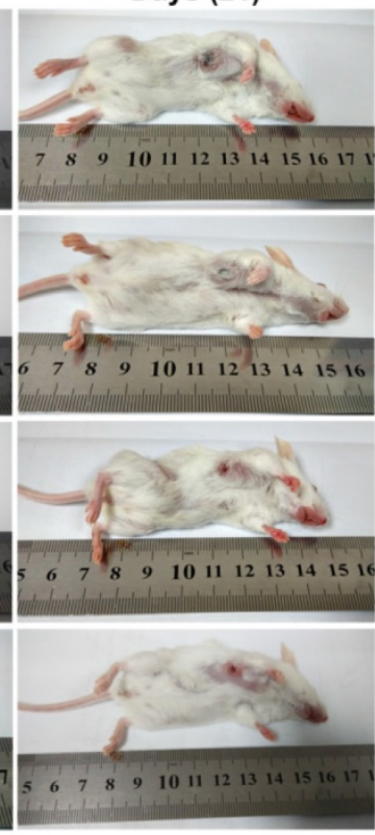

Microwave

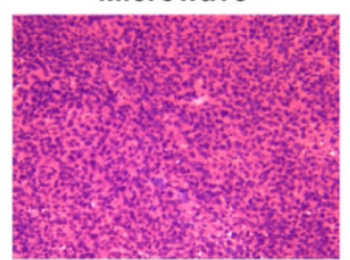

B
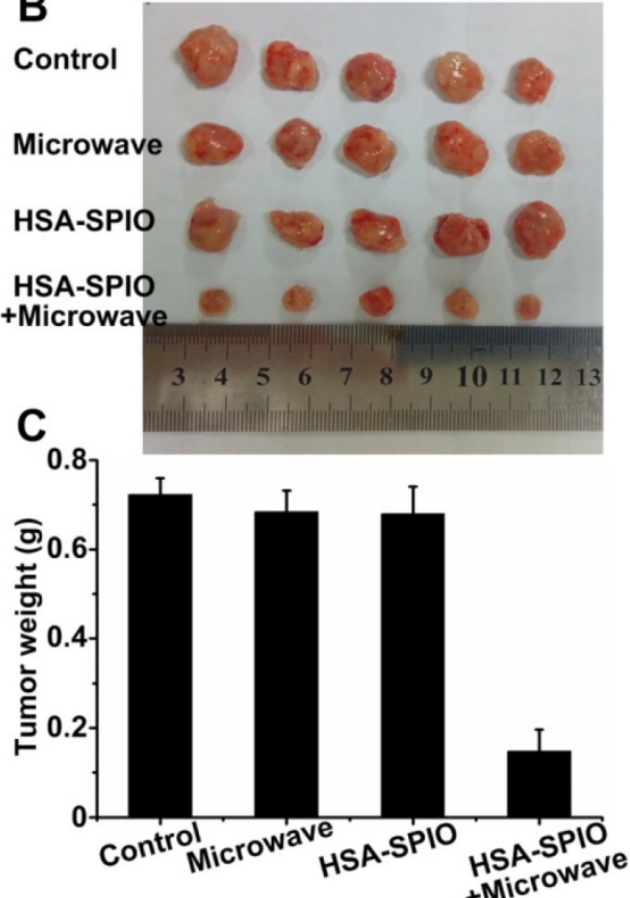

HSA-SPIO

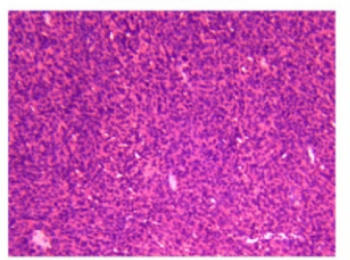

HSA-SPIO+Microwave

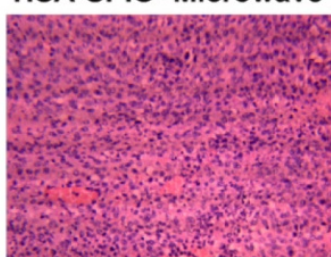

Figure 7. TA therapy in a deep-seated orthotopic tumor model. (A) Photographic images of different treatment mice (pre- and post-21 days of treatment). (B) Representative photographic images of tumors in different treatment groups at post-21 days; (C) Tumor weight in different treatment groups 21 days after treatment. Data presented as mean \pm SD $(n=5)$. (D) Representative H\&E-stained sections of tumor tissue from different treatment groups (10× magnification).

\section{TA therapy in orthotopic tumor model}

The excellent performance of HSA-SPIO in tumor accumulation, cell killing effect as well as the highly-efficient TA therapy in subcutaneous tumor models inspired us to further explore their potential application in deep-seated tumor models, given the deep penetrability of microwave in tissues. Orthotopic breast tumors were inoculated into the mammary fat pad of mice to establish a deep-seated tumor model. Mice photographs were recorded before TA treatment and 21 days later. Tumors in the HSA-SPIO+Microwave group were confined in situ and its growth was inhibited, while tumors in the other groups showed rapid growth (Fig. 7A). Mice were sacrificed 21 days later and the size/weights of tumors were compared (Fig. 7B). Mean tumor weight in the HSA-SPIO+Microwave group $(0.15 \pm 0.05 \mathrm{~g})$ was significantly lower than those in the control $(0.72$ $\pm 0.03 \mathrm{~g})$, microwave-alone $(0.68 \pm 0.05 \mathrm{~g})$ and HSA-SPIO-alone $(0.68 \pm 0.06 \mathrm{~g})$ groups (Fig. 7C). On histological examination, tumor sections from the
HSA-SPIO+Microwave group showed more vacuoles, condensed nuclei, and change in cell shapes consistent with severe injury, while no obvious cell destruction was observed in the other groups (Fig. 7D). All of the above results indicated that the TA effect of HSA-SPIO pumped by pulsed microwave could not only inhibit tumor growth in the subcutaneous xenograft tumor models but also in the deep-seated tumor models.

\section{Discussion}

Multifunctional nanoparticle-mediated microwave thermal therapy has been shown to be highly efficient in ablating tumors [53-58]. It shows great promising in cancer therapy, which combines the advantages of deep tissue penetrability with excellent safety and efficacy. In order to explore the potential of microwave therapy for clinical application, we developed a new cancer therapeutic approach named TA therapy, which is based on the thermoelastic effect of mitochondria-targeting single wall carbon nanotubes (SWNTs) pumped by pulsed microwave 
[32]. TA therapy differs from microwave thermal therapy in several respects. Firstly, the microwave generation source is different. The microwave thermal therapy used a continuous microwave source, while TA therapy employs ultra-short pulsed microwave. The pulse repetition frequency and pulse energy of ultra-short pulsed microwave is adjustable which was closely related to the treatment efficiency. Secondly, the microwave thermal therapy employs use of microwave susceptible agents such as ionic liquid to absorb the continuous microwave energy, which is then transferred to the kinetic and interionic energy. Thereafter, the energy is transformed into Joule heating of salt ions. The ionic liquid was encapsulated in the microcapsule, leading to more frequent interactions between salt ions, and rapid increase in temperature. In contrast, nanoagents such as mitochondria-targeting SWNTs absorb pulsed microwave energy and transform it into TA shockwave which causes selective destruction of the targeted mitochondria followed by apoptosis of cancer cells. Based on the theory of TA effect, the contrast agent absorbed pulsed microwave and converted into heat. The nanoagents are instantly heated and undergo rapid thermal expansion, and the energy is ultimately released in the form of TA shockwave [59]. Similar to the photoacoustic effect, once the microwave absorber absorbs a microwave pulse with a temporal duration of $\tau_{p}$, the thermal diffusion length during the pulse period can be estimated by $\delta_{T}=2 \sqrt{D_{T} \tau_{p}}$, where $D_{T}$ is the thermal diffusivity of the sample, and a typical value for most soft tissues was $\mathrm{D}_{\mathrm{T}} \approx 1.4 \times 10^{-3} \mathrm{~cm}^{2} / \mathrm{s}$ [60]. Given that microwave power dissipation in a region was uniform and so rapid, the ultra-short pulsed microwave $\left(\tau_{p}=10\right.$ ns) induced thermal diffusion in biological tissues would be negligible $\left(\delta_{\mathrm{T}} \approx 0.075 \mu \mathrm{m}\right)$ [61]. On the other hand, if the pulse width of the excited microwave is less than the thermal relaxation time, the instantaneous temperature rise in tissues is expressed by the equation $\Delta \mathrm{T}=\mathrm{E}_{\mathrm{a}} / \mathrm{C}_{\mathrm{p}} \rho$, where $\mathrm{E}_{\mathrm{a}}$ is the energy density per volume. As an example, the parameters for muscle tissue are $C_{p} \approx 3.7 \mathrm{~J} /(\mathrm{gK})$ and $\rho \approx 1.06$ $\mathrm{g} / \mathrm{cm}^{3}$. The nanosecond pulsed microwave with a 10 ns pulse width, energy density per area of $400 \mu \mathrm{J} / \mathrm{cm}^{2}$ and penetration depth of about $3.57 \mathrm{~cm}$ will result in an instantaneous temperature rise of $0.4 / 3.57 /(3.57 \times 1.06)=0.03 \mathrm{mK}$. Even on irradiation for $10 \mathrm{~min}$, the total temperature rise using a nanosecond pulsed microwave will be $0.18 \mathrm{~K}$, which can be almost ignored. Moreover, the thermoregulatory mechanism of the human body further reduces the temperature increment [62]. Based on these, it can be theoretically predicted that pulsed microwave hardly caused thermal damage. We believe that the TA therapeutic effect does not depend on hyperthermia, thus it did not cause damage to normal tissues or drug resistance. Lastly, the shockwave generated in TA therapy can be acquired and reconstructed to realize TA imaging. TA imaging with relative low energy microwave and low concentration of contrast agents affords greater resolution and contrast. It provides real-time information of change in TA signals and dynamic distribution of nanoagents in the tumor, which allows choosing the right time for the subsequent TA therapy. TA imaging shows good prospects for early detection of localization and to monitor dynamic distribution of nanoagents. Moreover, TA therapy induced by high doses of microwave and nanoagents showed evident anti-tumor effects for a deep-seated tumor model due to deep penetrability of microwaves in biological tissue. Therefore TA imaging and imaging guided therapy as a new theranostic technique is worthy of further research and development in a standard ultra-short pulsed microwave platform.

Our preliminary results show cell apoptosis to be the main mechanism of cell death caused by TA therapy. It is considered to be better for cancer cell elimination because apoptotic cells could afford more tumor antigens and induce high immune response [63]. We believe that the TA shockwave in TA therapy is essentially similar to the cavitation effect reported in photoacoustic therapy [4]. Pulsed microwave with $0.5 \mu$ s-pulse width was shown to induce TA effect on tissues by causing increase in pressure of the order of several hundred Pascals [64]. In this study, the absorption coefficient of HSA-SPIO as a microwave-absorbing agent was shown to be several times or even several dozen times higher than that of biological tissue at $434 \mathrm{MHz}[35,45,60]$, which would enhance the TA pressure on HSA-SPIO several dozen-fold higher than that on biological tissue. Moreover, the development of ultra-short pulsed microwave has shown that TA pressure pumped by pulsed microwave with a 10 ns-pulse width could be enhanced hundred-fold of that with $0.5 \mu$ s-pulse width [30]. Therefore, benefiting from the ultra-short pulsed microwave with 10 ns-pulse width and SPIO as a microwave-absorbing agent, the TA pressure of ultra-short pulsed microwave pumped SPIO would enhance several hundreds or even thousand-fold than microwave (0.5 $\mu$ s-pulse width) pumped tissues (hundreds Pascals) and could be up to $0.1 \mathrm{MPa}$, which was shown to be sufficient to cause cavitation and induce cell apoptosis [65]. Based on this, TA shockwave destroys the cell membrane, leads to influx of extracellular $\mathrm{Ca}^{2+}$ followed by degradation of the actin network, and produces a dramatic blebbing 
response [66]. Moreover, micro-explosion in lysosomes triggers release of lysosomal proteases, and activation of caspases, thereby inducing cell apoptosis [67]. Overall, TA shockwave probably causes mechanical destruction of the cell membrane or lysosomes, enhances cell permeability and triggers cell apoptosis $[46,47,66]$.

In this study, although some of large HSA-SPIO exceeded $200 \mathrm{~nm}$, HSA-SPIO was found to have a good safety profile as a nanoprobe and did not cause obvious cytotoxicity and in vivo toxicity, which indicate their excellent biocompatibility. Therefore, HSA-SPIO consisting of two safe components shows great potential to be a clinical theranostic agent. Firstly, the albumin layer prevents recognition by the reticuloendothelial system (RES) to a large extent, meanwhile increases stability in plasma and prolongs the circulation time, which is attributed to the protection conferred by the albumin layer and the more permeable microvascular environment of the tumor tissue, i.e. the EPR effect (enhanced permeability and retention effect). Furthermore, the interaction of the HSA sheath with cell surface glycoprotein (gp60) receptor (albondin) and/or SPARC (secreted protein acid and rich in cysteine) expressed on the surface of various types of cancer cells facilitates the transportation of the nanoparticles $[68,69]$. This targeted interaction also plays an important role in tumor accumulation and explains the excellent cellular uptake. In addition, significant magnetism of SPIO makes it a good MRI probe with the ability to realize magnetic targeting in future applications.

Microwave irradiation exposes a relatively large area of the body to microwave field. Protection of normal tissues from microwave-induced damage is essential to safe clinical application in future. In particular, prolonged accumulation of nanoagents in metabolically active organs such as liver and kidney is a key concern. Therefore, improving the targeting of nanoagents and reducing the microwave dose are two straight forward ways to minimize the potential side effects. Development of focused pulsed microwave has made treatment of specific solid tumors feasible with minimal damage to normal tissues. Moreover, covering of normal parts with metallized knitted net fabrics protects against microwave radiation. Further development of TA therapy aim at the specific tumor sites by designing different metallized knitted net fabrics clothes and radiating local tumor sites.

\section{Conclusion}

In this study, we for the first time attempted to develop HSA-SPIO as a theranostic agent for MRI and TA imaging guided therapy in an ultra-short pulsed microwave platform. HSA-SPIO comprising of two clinically used components shows great potential to be a multifunctional agent in clinical settings. It not only provided clear anatomical image on MRI, but also showed a high sensitivity for detection of nanoprobes accumulation and TA effect enhancement in tumor region, which provided comprehensive and complementary information of tumor. More importantly, HSA-SPIO mediated TA therapy achieved outstanding anti-tumor effect even for the deep-seated tumor model, because of the great microwave-absorbing properties of HSA-SPIO, ultra-short pulsed microwave with high energy conversion efficiency to excite shockwave and deep penetrability for biological tissue. Fortunately, the mechanism of TA therapy was based on the TA effect-induced cavitation and did not depend on hyperthermia, which avoid the thermal injury to the contiguous healthy tissues. Therefore, thermoelastic effect of multifunctional nanoprobes pumped by ultra-short pulsed microwave shows great promising for further development as a versatile technique for visualizing tumor localization, monitoring distribution of nanoprobes and highly-efficiently inhibiting tumor growth.

\section{Supplementary Material}

Supplementary materials and methods, Supplementary figures S1-S12.

http://www.thno.org/v07p1976s1.pdf

\section{Acknowledgments}

This research is supported by the National Natural Science Foundation of China (61627827; 81630046; 61331001; 91539127), the Science and Technology Planning Project of Guangdong Province, China (2015B020233016; 2014B020215003; 2014A020215031), the Distinguished Young Teacher Project in Higher Education of Guangdong, China (YQ2015049).

\section{Competing Interests}

The authors have declared that no competing interest exists.

\section{References}

1. Wei YC, Chen Q, Wu BY, Xing D. Excitation-Selectable Nanoprobe for Tumor Fluorescence Imaging and Near-Infrared Thermal Therapy. J Biomed Nanotechnol. 2016; 12: 91-102

2. Hong G, Robinson JT, Zhang $Y$, Diao S, Antaris AL, Wang Q, et al. In vivo fluorescence imaging with $\mathrm{Ag}_{2} \mathrm{~S}$ quantum dots in the second near-infrared region. Angew Chem Int Ed Engl. 2012; 51: 9818-21.

3. Jiang P, Zhu CN, Zhang ZL, Tian ZQ, Pang DW. Water-soluble Ag(2)S quantum dots for near-infrared fluorescence imaging in vivo. Biomaterials. 2012; 33: 5130-5.

4. Zhong J, Wen L, Yang S, Xiang L, Chen Q, Xing D. Imaging-guided high-efficient photoacoustic tumor therapy with targeting gold nanorods. Nanomedicine-Uk. 2015; 11: 1499-509. 
5. Zhong JP, Yang SH, Wen LW, Xing D. Imaging-guided photoacoustic drug release and synergistic chemo-photoacoustic therapy with paclitaxel-containing nanoparticles. J Control Release. 2016; 226: 77-87.

6. Qin H, Zhou T, Yang SH, Xing D. Fluorescence Quenching Nanoprobes Dedicated to In Vivo Photoacoustic Imaging and High-Efficient Tumor Therapy in Deep-Seated Tissue. Small. 2015; 11: 2675-86.

7. Cheng L, Yang K, Li YG, Zeng X, Shao MW, Lee ST, et al. Multifunctional nanoparticles for upconversion luminescence/MR multimodal imaging and magnetically targeted photothermal therapy. Biomaterials. 2012; 33: 2215-22.

8. Hu XL, Liu GH, Li Y, Wang XR, Liu SY. Cell-Penetrating Hyperbranched Polyprodrug Amphiphiles for Synergistic Reductive Milieu-Triggered Drug Release and Enhanced Magnetic Resonance Signals. J Am Chem Soc. 2015; 137: 362-8.

9. Hayashi K, Nakamura M, Sakamoto W, Yogo T, Miki H, Ozaki S, et al. Superparamagnetic Nanoparticle Clusters for Cancer Theranostics Combining Magnetic Resonance Imaging and Hyperthermia Treatment. Theranostics. 2013; 3: 366-76.

10. Pang B, Zhao Y, Luehmann H, Yang X, Detering L, You M, et al. (64)Cu-Doped PdCu@Au Tripods: A Multifunctional Nanomaterial for Positron Emission Tomography and Image-Guided Photothermal Cancer Treatment. ACS Nano. 2016; 10: 3121-31.

11. Gao FP, Cai PJ, Yang WJ, Xue JQ, Gao L, Liu R, et al. Ultrasmall [Cu-64] Cu Nanoclusters for Targeting Orthotopic Lung Tumors Using Accurate Positron Emission Tomography Imaging. ACS Nano. 2015; 9: 4976-86.

12. Hu H, Huang P, Weiss OJ, Yan XF, Yue XY, Zhang MG, et al. PET and NIR optical imaging using self-illuminating Cu-64-doped chelator-free gold nanoclusters. Biomaterials. 2014; 35: 9868-76.

13. Deng H, Zhong Y, Du M, Liu Q, Fan Z, Dai F, et al. Theranostic self-assembly structure of gold nanoparticles for NIR photothermal therapy and X-Ray computed tomography imaging. Theranostics. 2014; 4: 904-18.

14. Ni DL, Zhang JW, Bu WB, Zhang $C$, Yao ZW, Xing HY, et al. PEGylated $\mathrm{NaHoF}_{4}$ nanoparticles as contrast agents for both X-ray computed tomography and ultra-high field magnetic resonance imaging. Biomaterials. 2016; 76: 218-25.

15. Zhou FF, Wu SN, Wu BY, Chen WR, Xing D. Mitochondria-Targeting Single-Walled Carbon Nanotubes for Cancer Photothermal Therapy. Small. 2011; 7: 2727-35.

16. Chen Q, Liang C, Wang C, Liu Z. An Imagable and Photothermal "Abraxane-Like" Nanodrug for Combination Cancer Therapy to Treat Subcutaneous and Metastatic Breast Tumors. Adv Mater. 2015; 27: 903-10.

17. Zhou FF, Wu SN, Yuan Y, Chen WR, Xing D. Mitochondria-Targeting Photoacoustic Therapy Using Single-Walled Carbon Nanotubes. Small. 2012; 8: $1543-50$

18. Zhang Y, Qian J, Wang D, Wang Y, He S. Multifunctional gold nanorods with ultrahigh stability and tunability for in vivo fluorescence imaging, SERS detection, and photodynamic therapy. Angew Chem Int Ed Engl. 2013; 52: 1148-51.

19. Kalluru P, Vankayala R, Chiang CS, Hwang KC. Nano-graphene oxide-mediated In vivo fluorescence imaging and bimodal photodynamic and photothermal destruction of tumors. Biomaterials. 2016; 95: 1-10.

20. Jang B, Park JY, Tung CH, Kim IH, Choi Y. Gold nanorod-photosensitizer complex for near-infrared fluorescence imaging and photodynamic/photothermal therapy in vivo. ACS Nano. 2011; 5: 1086-94.

21. Espinosa A, Di Corato R, Kolosnjaj-Tabi J, Flaud P, Pellegrino T, Wilhelm C. Duality of Iron Oxide Nanoparticles in Cancer Therapy: Amplification of Heating Efficiency by Magnetic Hyperthermia and Photothermal Bimodal Treatment. ACS Nano. 2016; 10: 2436-46.

22. Xu CY, Zheng $Y Y$, Gao W, Xu JS, Zuo GQ, Chen $Y$, et al. Magnetic Hyperthermia Ablation of Tumors Using Injectable $\mathrm{Fe}_{3} \mathrm{O}_{4} /$ Calcium Phosphate Cement. ACS Appl Mater Interfaces. 2015; 7: 13866-75.

23. Huynh E, Lovell JF, Helfield BL, Jeon M, Kim C, Goertz DE, et al. Porphyrin Shell Microbubbles with Intrinsic Ultrasound and Photoacoustic Properties. J Am Chem Soc. 2012; 134: 16464-7.

24. Huang JW, Xu JS, Xu RX. Heat-sensitive microbubbles for intraoperative assessment of cancer ablation margins. Biomaterials. 2010; 31: 1278-86.

25. Liu J, Yang Y, Zhu W, Yi X, Dong Z, Xu X, et al. Nanoscale metal-organic frameworks for combined photodynamic \& radiation therapy in cancer treatment. Biomaterials. 2016; 97: 1-9.

26. Cheng L, Shen SD, Shi SX, Yi Y, Wang XY, Song GS, et al. $\mathrm{FeSe}_{2}$-Decorated $\mathrm{Bi}_{2} \mathrm{Se}_{3}$ Nanosheets Fabricated via Cation Exchange for Chelator-Free Cu-64-Labeling and Multimodal Image-Guided Photothermal-Radiation Therapy. Adv Funct Mater. 2016; 26: 2185-97.

27. Pearce JA, Cook JR, Emelianov SY. Ferrimagnetic Nanoparticles Enhance Microwave Heating for Tumor Hyperthermia Therapy. IEEE Eng Med Bio. 2010: 2751-4.

28. Ku G, Fornage BD, Jin $\mathrm{X}, \mathrm{Xu} \mathrm{M}$, Hunt KK, Wang LV. Thermoacoustic and photoacoustic tomography of thick biological tissues toward breast imaging. Technol Cancer Res Treat. 2005; 4: 559-66.

29. Ji Z, Lou C, Yang S, Xing D. Three-dimensional thermoacoustic imaging for early breast cancer detection. Med Phys. 2012; 39: 6738-44.

30. Lou C, Yang S, Ji Z, Chen Q, Xing D. Ultrashort microwave-induced thermoacoustic imaging: a breakthrough in excitation efficiency and spatial resolution. Phys Rev Lett. 2012; 109: 218101.
31. Feng D, Xu Y, Ku G, Wang LV. Microwave-induced thermoacoustic tomography: reconstruction by synthetic aperture. Med Phys. 2001; 28: 2427-31.

32. Wen L, Ding W, Yang S, Xing D. Microwave pumped high-efficient thermoacoustic tumor therapy with single wall carbon nanotubes. Biomaterials. 2016; 75: 163-73.

33. Elzoghby AO, Samy WM, Elgindy NA. Albumin-based nanoparticles as potential controlled release drug delivery systems. J Control Release. 2012; 157: $168-82$

34. Gause KT, Yan Y, Cui JW, O'Brien-Simpson NM, Lenzo JC, Reynolds EC, et al. Physicochemical and Immunological Assessment of Engineered Pure Protein Particles with Different Redox States. ACS Nano. 2015; 9: 2433-44.

35. Xie J, Chen K, Huang J, Lee S, Wang JH, Gao J, et al. PET/NIRF/MRI triple functional iron oxide nanoparticles. Biomaterials. 2010; 31: 3016-22.

36. Cheng FY, Su CH, Yang YS, Yeh CS, Tsai CY, Wu CL, et al. Characterization of aqueous dispersions of $\mathrm{Fe}_{3} \mathrm{O}_{4}$ nanoparticles and their biomedical applications. Biomaterials. 2005; 26: 729-38.

37. Kim DK, Amin MS, Elborai S, Lee SH, Koseoglu Y, Muhammed M, et al. Energy absorption of superparamagnetic iron oxide nanoparticles by microwave irradiation. J Appl Phys. 2005; 97.

38. Ni SB, Lin SM, Pan QT, Yang F, Huang K, He DY. Hydrothermal synthesis and microwave absorption properties of $\mathrm{Fe}_{3} \mathrm{O}_{4}$ nanocrystals. J Phys D Appl Phys. $2009 ; 42$.

39. Nie LM, Ou ZM, Yang SH, Xing D. Thermoacoustic molecular tomography with magnetic nanoparticle contrast agents for targeted tumor detection. Med Phys. 2010; 37: 4193-200.

40. Zhou T, Wu BY, Xing D. Bio-modified $\mathrm{Fe}_{3} \mathrm{O}_{4}$ core/Au shell nanoparticles for targeting and multimodal imaging of cancer cells. J Mater Chem. 2012; 22: 470-7.

41. Jin X, Keho A, Meissner K, Wang LV. Iron oxide nanoparticles as a contrast agent in thermoacoustic tomography - art. no. 64370E. P Soc Photo-Opt Ins. 2007; 6437: E4370-E.

42. Liu J, Sun ZK, Deng YH, Zou Y, Li CY, Guo XH, et al. Highly Water-Dispersible Biocompatible Magnetite Particles with Low Cytotoxicity Stabilized by Citrate Groups. Angew Chem Int Ed Engl. 2009; 48: 5875-9.

43. Ku G, Wang LHV. Scanning microwave-induced thermoacoustic tomography: Signal, resolution, and contrast. Med Phys. 2001; 28: 4-10.

44. Ji Z, Lou CG, Yang SH, Xing D. Three-dimensional thermoacoustic imaging for early breast cancer detection. Med Phys. 2012; 39: 6738-44.

45. Johnson CC, Guy AW. Nonionizing Electromagnetic Wave Effects in Biological Materials and Systems. Proceedings of The IEEE, VOL. 1972; 60: 6.

46. Kang B, Yu DC, Dai YD, Chang SQ, Chen D, Ding YT. Cancer-Cell Targeting and Photoacoustic Therapy Using Carbon Nanotubes as "Bomb" Agents. Small. 2009; 5: 1292-301.

47. Chen CL, Kuo LR, Chang CL, Hwu YK, Huang CK, Lee SY, et al. In situ real-time investigation of cancer cell photothermolysis mediated by excited gold nanorod surface plasmons. Biomaterials. 2010; 31: 4104-12.

48. Harrison RA, Vickers SE. Use of fluorescent probes to assess membrane integrity in mammalian spermatozoa. J Reprod Fertil. 1990; 88: 343-52.

49. Buja LM, Eigenbrodt ML, Eigenbrodt EH. Apoptosis and necrosis. Basic types and mechanisms of cell death. Arch Pathol Lab Med. 1993; 117: 1208-14.

50. Miele E, Spinelli GP, Miele E, Tomao F, Tomao S. Albumin-bound formulation of paclitaxel (Abraxane (R) ABI-007) in the treatment of breast cancer. Int J Nanomed. 2009; 4: 99-105.

51. Thorek DLJ, Chen A, Czupryna J, Tsourkas A. Superparamagnetic iron oxide nanoparticle probes for molecular imaging. Ann Biomed Eng. 2006; 34: 23-38.

52. Song XJ, Gong H, Yin SN, Cheng L, Wang C, Li ZW, et al. Ultra- Small Iron Oxide Doped Polypyrrole Nanoparticles for In Vivo Multimodal Imaging Guided Photothermal Therapy. Adv Funct Mater. 2014; 24: 1194-201.

53. Shi HT, Niu M, Tan LF, Liu TL, Shao HB, Fu CH, et al. A smart all-in-one theranostic platform for CT imaging guided tumor microwave thermotherapy based on IL@ZrO ${ }_{2}$ nanoparticles. Chem Sci. 2015; 6: 5016-26.

54. Shi HT, Liu TL, Fu CH, Li LL, Tan LF, Wang JZ, et al. Insights into a microwave susceptible agent for minimally invasive microwave tumor thermal therapy. Biomaterials. 2015; 44: 91-102.

55. Tang WT, Liu B, Wang SP, Liu TL, Fu CH, Ren XL, et al. Doxorubicin-loaded ionic liquid-polydopamine nanoparticles for combined chemotherapy and microwave thermal therapy of cancer. Rsc Adv. 2016; 6: 32434-40.

56. Du QJ, Fu CH, Tie J, Liu TL, Li LL, Ren XL, et al. Gelatin microcapsules for enhanced microwave tumor hyperthermia. Nanoscale. 2015; 7: 3147-54.

57. Du QJ, Ma TC, Fu CH, Liu TL, Huang ZB, Ren J, et al. Encapsulating Ionic Liquid and $\mathrm{Fe}_{3} \mathrm{O}_{4}$ Nanoparticles in Gelatin Microcapsules as Microwave Susceptible Agent for MR Imaging-guided Tumor Thermotherapy. ACS Appl Mater Interfaces. 2015; 7: 13612-9.

58. Tan LF, Tang WT, Liu TL, Ren XL, Fu CH, Liu B, et al. Biocompatible Hollow Polydopamine Nanoparticles Loaded Ionic Liquid Enhanced Tumor Microwave Thermal Ablation in Vivo. ACS Appl Mater Interfaces. 2016; 8: 11237-45.

59. Ji $\mathrm{Z}, \mathrm{Fu} \mathrm{Y}$, Yang $\mathrm{SH}$. Microwave-Induced Thermoacoustic Imaging for Early Breast Cancer Detection. J Innov Opt Heal Sci. 2013; 6: 1350001.

60. Xu MH, Wang LHV. Photoacoustic imaging in biomedicine. Rev Sci Instrum. 2006; 77: 041101

61. Mashal A, Booske JH, Hagness SC. Toward contrast-enhanced microwave-induced thermoacoustic imaging of breast cancer: an experimental 
study of the effects of microbubbles on simple thermoacoustic targets. Phys Med Biol. 2009; 54: 641-50.

62. Ye FH, Ji Z, Ding WZ, Lou CG, Yang SH, Xing D. Ultrashort Microwave-Pumped Real-Time Thermoacoustic Breast Tumor Imaging System. IEEE Trans Med Imaging. 2016; 35: 839-44.

63. Zhou FF, Xing D, Chen WR. Regulation of HSP70 on activating macrophages using PDT-induced apoptotic cells. Int J Cancer. 2009; 125: 1380-9.

64. Xu MH, Wang LHV. Pulsed-microwave-induced thermoacoustic tomography: Filtered backprojection in a circular measurement configuration. Med Phys. 2002; 29: 1661-9.

65. Feril LB, Jr., Kondo T, Cui ZG, Tabuchi Y, Zhao QL, Ando H, et al. Apoptosis induced by the sonomechanical effects of low intensity pulsed ultrasound in a human leukemia cell line. Cancer Lett. 2005; 221: 145-52.

66. Tong L, Zhao Y, Huff TB, Hansen MN, Wei A, Cheng JX. Gold Nanorods Mediate Tumor Cell Death by Compromising Membrane Integrity. Adv Mater. 2007; 19: 3136-41.

67. Boya P, Kroemer G. Lysosomal membrane permeabilization in cell death. Oncogene. 2008; 27: 6434-51.

68. Desai N, Trieu V, Yao Z, Louie L, Ci S, Yang A, et al. Increased antitumor activity, intratumor paclitaxel concentrations, and endothelial cell transport of cremophor-free, albumin-bound paclitaxel, ABI-007, compared with cremophor-based paclitaxel. Clin Cancer Res. 2006; 12: 1317-24.

69. Porter PL, Sage EH, Lane TF, Funk SE, Gown AM. Distribution of SPARC in normal and neoplastic human tissue. J Histochem Cytochem. 1995; 43: 791-800. 